\title{
HIV Second hideout for HIV-1
}

Immature multipotent haematopoietic progenitor cells (HPCs) are a second reservoir of latent HIV-1 infection, according to a new report in Nature Medicine.

Although highly active antiretroviral therapy (HAART) has greatly reduced mortality from HIV-1 infection, it cannot completely eradicate the virus owing to viral persistence as a provirus in latently infected cells. The main cellular reservoir for latent $\mathrm{HIV}-1$ infection is resting $\mathrm{CD} 4^{+}$ $\mathrm{T}$ cells, and the biology of latency in these cells has been intensively investigated. However, the existence of other cellular reservoirs

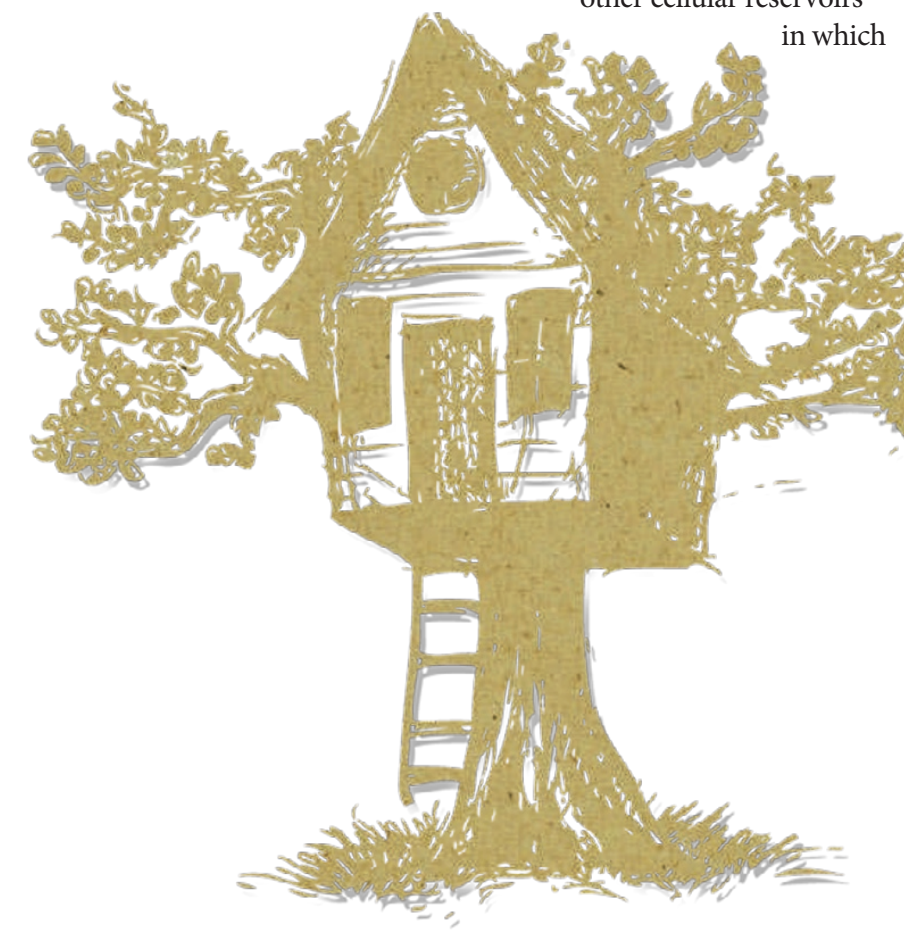

residual virus replication occurs has long been suspected, with a key candidate being HPCs. These CD34 bone marrow cells comprise a heterogeneous mixture of haematopoietic stem cells (HSCs) and multipotent progenitor cells that form as a result of HSC self-renewal and proliferation.

Collins and colleagues analysed the susceptibility of HPCs to HIV-1 infection and found that the virus can actively infect multipotent progenitor cells and cause cell death. But can HIV-1 establish a latent infection in these cells? Direct detection of latent infection involved the creation of a reporter virus expressing GFP independently of the HIV-1 long terminal repeat; this allows latently infected cells to be detected, as they express GFP but not viral proteins such as Gag. Distinct populations of actively infected $\left(\mathrm{Gag}^{+} \mathrm{GFP}^{+}\right)$and latently infected $\left(\mathrm{Gag}^{-} \mathrm{GFP}^{+}\right) \mathrm{HPCs}$ were detected, and a detailed flow cytometry analysis of the cell surface markers present on the latently infected cells revealed that they were immature multipotent HPCs.

To check whether latent HIV-1 could be reactivated from the HPC reservoir, the authors used the stimulator phorbol 12-myristate 13-acetate (PMA) to induce cellular differentiation of bone marrow-derived HPCs that were infected with HIV-1 expressing a marker protein. PMA treatment led to a large increase in the production of virus particles and expression of the marker protein compared with a control treatment. In addition, when bone marrow-derived HPCs infected with HIV-1 were treated with the cytokines granulocyte-macrophage colony-stimulating factor and tumour necrosis factor, which stimulate HPC differentiation into the myeloid lineage, there was a rapid release of HIV-1 into the culture supernatant. Finally, the authors detected $\mathrm{CD} 4^{+} \mathrm{Gag}^{+}$cells in six HIV-1-infected individuals with high viral loads, and they detected HIV genomes in four out of nine individuals who had been receiving HAART for at least 6 months and in whom the viral load was undetectable.

So, in addition to the reservoir in $\mathrm{CD} 4^{+} \mathrm{T}$ cells, it seems that HIV-1 can persist in immature multipotent HPCs. Further work is required to investigate whether the virus is also present in $\mathrm{CD}_{3}{ }^{+} \mathrm{HSC}$ and whether this HPC reservoir is the source of the residual viraemia that is observed in individuals receiving HAART.

Sheilagh Molloy

ORIGINAL RESEARCH PAPER Carter, C. C. et al. HIV-1 infects multipotent progenitor cells causing cell death and establishing latent cellular reservoirs. Nature Med. 10 Mar2010 (doi: 10.1038/ nm.2109)

FURTHER READING Han, Y. et al. Experimental approaches to the study of HIV-1 latency. Nature Rev. Microbiol. 5, 96-106 (2007) 